\title{
Teaching Mental Health First Aid in the school setting:
}

\section{a novel approach to improving outcomes for common adolescent}

\section{mental disorders.}

\author{
Laura M Hart $^{1,2 \infty}$, Georgina R Cox ${ }^{3}$, Lucy Lees ${ }^{1}$
}

1 Centre for Mental Health, Melbourne School of Population and Global Health, University of Melbourne, Australia

2 School of Psychology and Public Health, La Trobe University, Australia

3 Orygen The National Centre of Excellence in Youth Mental Health

${ }^{\infty}$ Corresponding author: 1.hart@unimelb.edu.au +61 39479 6495, 207 Bouverie Street Carlton, 3010. 


\section{Abstract}

Purpose of review: Common adolescent mental disorders, such as depression, often go untreated and severely impact health and educational outcomes. The purpose of this review is to describe what is currently known about school-based mental health interventions and to describe a new intervention; Mental Health First Aid training.

Recent findings: Universal and selective prevention and treatment programs have been widely evaluated, though population-level dissemination remains elusive. A novel approach is to train adolescents in how to recognise early signs of mental disorder onset, decrease stigmatising beliefs and barriers to help-seeking, and to use appropriate first aid strategies for assisting peers in mental health crisis, such as those with depression and suicidal ideation.

Summary: Teaching adolescents the skills necessary to recognise and respond to mental health problems and mental health crises may provide life-long skills that prompt lower stigmatizing beliefs, greater support of peers and appropriate, timely help-seeking. [147 words]

Keywords: mental health first aid, depression, help-seeking, adolescents, school. 


\section{Introduction}

Adolescence is the peak period for the onset of many mental illnesses (1). In particular, depression commonly emerges during the high school years, and is among the leading causes of burden of disease in young females and males (2). By age 16, 11.7\% of females and $7.3 \%$ of males are estimated to have experienced a depressive episode (3) the symptoms of which can negatively impact school performance, relationships with friends and family, and in more severe cases, lead to suicidal ideation and attempts (4).

Despite the high risk of depression recurrence and relapse into adulthood (5); a significant proportion of young people fail to seek help or receive treatment during this critical developmental period (6). Among American adolescents with symptoms of depression, treatment-seeking is reported by only $40 \%$ of females and $36 \%$ of males (7). Only one third of adolescents with any mental disorder are estimated to receive treatment (7).

\section{School based mental health interventions}

Schools are an important base for teaching children and adolescents about mental health and the last 20 years has seen the introduction of seminal school-based programs. Recent systematic reviews of school-based interventions report that a wide range of mental health programs have been trialled, across students of all age ranges, and with various health promotion, disorder prevention, and symptom-reduction therapeutic modalities (8, 9).

Universal promotion programs, such as the Beyondblue Schools Research Initiative (10) and Mindmatters (11), are delivered to all students and commonly aim to increase emotional resilience. Many utilize a cognitive behavioural therapy (CBT) framework, teaching young people to identify and reframe unhelpful thought patterns, and identify and 
engage in pleasant activities (12). Selective interventions have also been delivered in a school setting (9), however these are delivered only to those at high risk for a particular disorder. They commonly screen for the presence of symptoms and provide some components of therapy or treatment referral to reduce risk of transition to clinical disorder.

Recent reviews of school-based depression programs specifically suggest that there is benefit to their implementation $(8,13)$, though there are important barriers that need be overcome. First, the most effective programs are selective rather than universal, meaning that they require screening tools, psychometric interpretation, and are delivered only to atrisk rather than all students. Second, intervention effects are maximised when the programs are presented by trained health professionals (e.g. psychologists) rather than school staff (13). Providing curriculum-based program materials to regular classroom teachers, without specialist training in mental health or structural support from school leadership, may leave teachers feeling too inexperienced and overwhelmed to enact interventions with high fidelity $(10,14)$. Indeed cost-effectiveness analyses have concluded that school-based CBT programs are "unlikely to be either more effective or less costly than usual school provision" (p.1390, 15[DG1]) because of the need to employ health professionals or provide extensive training and support to regular classroom teachers. Third, most programs do not address barriers to treatment-seeking beyond the school environment (9) and thus may have restricted utility outside of school.

\section{Mental health first aid as a school-based intervention}

An innovative school-based mental health intervention is Mental Health First Aid training. Mental health first aid is defined as the help provided to a person developing a mental health problem or experiencing a mental health crisis. The first aid is given until the appropriate professional treatment is received, or the crisis resolves (16). Mental health first 
aid techniques have been taught in training programs offered by the Mental Health First Aid (MHFA) program since 2001. A meta-analysis of fifteen separate evaluations of MHFA training found that it is effective in improving knowledge, attitudes and behavioural intentions related to mental disorder, with the largest gains seen in improvements to knowledge (17).

Although the original version of MHFA training was designed to teach adults how to assist other adults with mental health problems, a tailored and specific version has been developed for adults assisting young people. The Youth Mental Health First Aid (YMHFA) course was launched in Australia in 2007 and has since been evaluated, updated through four editions (16), and disseminated throughout Europe and North America. YMHFA was launched as an 8-hour course in the United States in 2008 and has since undergone evaluations in various settings. Among graduate social work students (18), and a government social services department (19) YMHFA was associated with increased knowledge of mental disorders, problem recognition, confidence in providing help, and increased supportive first aid behaviours towards those experiencing a mental health problem. Although these changes were found among the adults attending training, neither study assessed the impact on adolescents. An Australian evaluation of the 16-hour YMHFA version implemented in a school setting found positive impacts on teachers' knowledge and confidence in providing help to students with mental health problems, as well as reductions in stgmatising attitudes, all of which were sustained over a 6 month period (20). Although students reported receiving more mental health information from school staff, no changes in student mental health or help-seeking were found.

In light of these findings, a specifically adapted program for students in the senior high school years was developed, with the aim of increasing knowledge and confidence, decrease stigmatising attitudes, and improving supportive first aid behaviours among peers 
$(21,22)$. teen Mental Health First Aid (tMHFA) is a three session classroom-based training program for students aged 15 to 18 years, which focuses on developing knowledge and skills in: recognising warning signs that a peer is developing a mental health problem, understanding how to talk to a peer about mental health and seeking help, when and how to tell a responsible adult, where to find appropriate and helpful resources about mental disorders and professional help, and how to respond in a crisis situation.

An uncontrolled pilot trial of tMHFA was conducted in 2012 (23) across four secondary schools in Australia, involving 988 students aged 15-17 years. Statistically significant improvements in knowledge and confidence when providing MHFA to a peer, plus decreases in stigmatising attitudes, were found over time. Importantly, although decreasing psychological distress was not a specific aim of the trial, a statistically significant reduction at follow-up was found on the K6 measure of psychological distress (24), despite this assessment coinciding with the peak exam period during the school year (23). The K6 is a short scale often used for population-level screening with higher scores indicating greater risk; it is able to correctly predict depression in $81-85 \%$ of cases, (25). Hence, a reduction in K6 scores may suggest the benefits of MHFA go beyond the first aid skills taught and towards positively impacting on student mental health.

More recently, a randomised control trial (RCT) involving a further four schools and 1,942 students has been conducted, comparing student outcomes before and after receiving either tMHFA or a matched emergency physical first aid (PFA) training program (26). Students who received tMHFA, compared to students receving PFA training, reported significantly more helpful MHFA intentions, less harmful MHFA intentions, increased confidence, lower stigmatising attitudes and more helpful beliefs about professional helpseeking, in response to brief vignettes describing fictional adolescent characters; one depicting depression and suicidal ideation, another depicting social phobia. Students 
receiving tMHFA were significantly more likely to indicate helpful first aid intentions by endorsing actions such as listening non-judgmentally and suggesting their friend get an adult involved, and significantly less likely to endorse 'harmful' furst aid intentions such as ignoring the person or doing nothing.

\section{Benefits of mental health first aid in the school setting}

Evaluation research of school-based MHFA training for adolescents is in its infancy, however, there are theoretical and practical reasons for its benefit, including: 1. a universal focus with scope for generating cultural change; 2 . bestowing behavioral skills on students that are highly likely to be enacted; and 3. decreasing school implementation barriers.

MHFA training focuses on improving knowledge of mental disorders and skills in supporting a peer with any type of mental health problem or mental health crisis and is delivered in a universal format to all students. School staff and parents are also encouraged to attend the YMHFA program to learn how to effectively support adolescents as their mental health literacy and help-seeking increases. This whole-of-school approach encourages cultural change, especially in reducing stigmatising attitudes and improving appropriate treatment-seeking. Although other universal programs to increase depression literacy have been trialled in US secondary schools (27), tMHFA is unique in its transdiagnostic focus and integrated whole-school approach. The recent RCT found that students who received tMHFA, compared to those receiving PFA, were twice as likely to recognise signs of suicidality in a peer, 46 times more likely to ask a suicidal peer if they were thinking about suicide, and 3.5 times more likely to speak to a peer about seeking help from an adult, after receiving their training (28). Previous tMHFA research has also shown that students report more favorable attitudes towards seeking help from teachers (23), and 
when teachers are MHFA trained they are more confident in addressing mental health concerns with students (20). This focus on social support is likely to be especially beneficial for students experiencing depression $(29,30)$, as their symptoms may be mitigated through high quality friendships. Indeed a strong benefit of MHFA training is to increase early recognition, effective and appropriate early intervention, and supportive social networks, which are all precipitating and maintaining factors for mental disorders (31).

Another benefit to MHFA training in schools is that it teaches behavioral skills and improves knowledge from the perspective of helping peers rather than the self. This perspective may engage the protégé phenomenon, where learners engage more deeply with topics when they have to teach material to others, as would be required when students enact MHFA and discuss the steps of seeking out help with a peer (32). MHFA skills have also been reported as more likely to be used than physical first aid strategies (33), and this is likely very true for adolescents who prefer to seek initial support from their peers . A large RCT is currently being conducted in Australia (see www.anzctr.org.au, ACTRN12614000061639) with student follow-up over 18-months, to investigate how prevalent the use of first aid skills are post-training, and to understand whether and how social interactions with peers lead to increased professional help-seeking and ultimately better mental health outcomes.

A third benefit of MHFA training is the reduced school implementation barriers. MHFA training began in Australia in 2001 but has since spread to more than 24 other countries, including the United States, Canada, England, Ireland, and others (34). The program uses a highly sustainable, structured, and thoroughly evaluated, train-the-trainer program (33), developed for school wellbeing staff (e.g., school counsellors or nurses) or appropriate health professionals (e.g., psychologists, social workers) to deliver the program 
within the school environment. Trainer-training is currently available in Australia and Ireland, but is also due for launch in Canada, England and potentially the USA in the next 12-months. The existing experience of MHFA organizations around the world mean that there is already a highly structured system of support for adequately training school staff and assisting them to implement the program with high fidelity. Alternatively, this model allows individual health professionals to act as private contractors to schools who do not have the capacity for their own staff to undergo trainer-training. Further research is required to fully understand the impact of providing adolescents with MHFA, but the benefits of improving mental health literacy within a whole school community appear to be clear.

\section{Conclusion}

Mental disorders such as depression are common in the adolescent years and there is need to increase appopriate help-seeking among teenagers. MHFA training aims to increase knowledge of mental disorders while decreasing stigmatising attitudes and barriers to helpseeking. Providing adolescents with MHFA training in the school setting appears to be a new and effective method for encouraging adolescents to use appropriate first aid strategies for assisting peers with a mental health problem, such as depression or suicidal ideation. [1,983 words] 


\section{Key Points}

- Common mental illnesses such as depression often emerge in adolescence, though only a minority of young people seek early and appropriate treatment.

- School-based mental health interventions have some evidence to suggest they are effective at reducing risk of disorders such as depression, if they are selective rather than universal and provided by trained health professionals rather than teachers without extra curriculum support.

- Mental Health First Aid training is designed to increase knowledge of mental disorders, improve supportive first aid behaviors, and encourage early and appropriate help seeking.

- Providing secondary students with Mental Health First Aid training is a novel school-based intervention which may increase peer support and facilitate help seeking for common adolescent mental disorders, such as depression. 


\section{References}

1. Kessler RC, Berglund P, Demler O, Jin R, Merikangas KR, Walters EE. Lifetime prevalence and age-of-onset distributions of DSM-IV disorders in the National Comorbidity Survey Replication. Archives of General Psychiatry. 2005;62(7):593-602. 2. Gore FM, Bloem PJN, Patton GC, Ferguson J, Joseph V, Coffey C, et al. Global burden of disease in young people aged 10-24 years: a systematic analysis. The Lancet. 2011;377(9783):2093-102.

3. Costello E, Mustillo S, Erkanli A, Keeler G, Angold A. Prevalence and development of psychiatric disorders in childhood and adolescence. Archives of General Psychiatry. 2003;60(8):837-44.

4. Thapar A, Collishaw S, Pine DS, Thapar AK. Depression in adolescence. The Lancet. 2012;379(9820):1056-67.

5. Peters AT, Jacobs RH, Feldhaus C, Henry DB, Albano AM, Langenecker SA, et al. Trajectories of functioning into emerging adulthood following treatment for adolescent depression. Journal of Adolescent Health. 2016;58(3):253-9.

6. Costello EJ, He J, Sampson NA, Kessler RC, Merikangas KR. Services for adolescents with psychiatric disorders: 12-month data from the National Comorbidity Survey-Adolescent. Psychiatric Services. 2014;65(3):359-66.

7. Merikangas KR, He J, Burstein M, Swendsen J, Avenevoli S, Case B, et al. Service utilization for lifetime mental disorders in U.S. adolescents: results of the National Comorbidity Survey-Adolescent Supplement (NCS-A). Journal of the American Academy of Child \& Adolescent Psychiatry. 2011;50(1):32-45.

* 8. Hetrick SE, Cox GR, Merry SN. Where to go from here? An exploratory metaanalysis of the most promising approaches to depression prevention programs for children 
and adolescents. International Journal Of Environmental Research And Public Health. 2015;12(5):4758-95.

This article highlights the range of interventions that have been evaluated in the school setting to prevent depression in adolescents.

** 9. Fazel M, Hoagwood K, Stephan S, Ford T. Mental health interventions in schools in high-income countries. The Lancet Psychiatry. 2014;1(5):377-87.

This article provides a comprehensive review of universal and selective interventions that have been evaluated in the school setting, to prevent or treat mental disorders in adolescents.

10. Sawyer MG, Harchak TF, Spence SH, Bond L, Graetz B, Kay D, et al. Schoolbased prevention of depression: a 2-Year follow-up of a randomized controlled trial of the beyondblue schools research initiative. Journal of Adolescent Health. 2010;47(3):297-304. 11. Rowling L. School mental health promotion: MindMatters as an example of mental health reform. Health Promotion Journal of Australia. 2007;18(3):229-35.

12. Rasing SPA, Creemers DHM, Janssens J, Scholte RHJ. Depression and Anxiety Prevention Based on Cognitive Behavioral Therapy for At-Risk Adolescents: A MetaAnalytic Review. Frontiers in psychology. 2017;8:1066.

* 13. Werner-Seidler A, Perry Y, Calear AL, Newby JM, Christensen H. School-based depression and anxiety prevention programs for young people: A systematic review and meta-analysis. Clinical psychology review. 2017;51:30-47.

This article provides the most recent meta-analysis of interventions evaluated in the school setting to prevent depression and anxiety in adolescents.

14. Han SS, Weiss B. Sustainability of teacher implementation of school-based mental health programs. Journal of Abnormal Child Psychology. 2005;33(6):665-79. 
15. Anderson R, Ukoumunne OC, Sayal K, Phillips R, Taylor JA, Spears M, et al. Costdepfectiveness of classroom-based,cognitive behaviour therapy in reducing symptoms of Psychiatry. 2014;55(12):1390-7.

16. Kelly CM, Kitchener BA, Jorm AF. Youth Mental Health First Aid: A manual for adults assisting young people. 4th ed. Melbourne, VIC: Mental Health First Aid Australia; 2017.

** 17. Hadlaczky G, Hökby S, Mkrtchian A, Carli V, Wasserman D. Mental Health First Aid is an effective public health intervention for improving knowledge, attitudes, and behaviour: A meta-analysis. International Review of Psychiatry. 2014;26(4):467-75. This is article provides a meta-analysis of 15 different studies evaluating Mental Health First Aid training.

18. Rose T, Leitch J, Collins KS, Frey JJ, Osteen PJ. Effectiveness of Youth Mental Health First Aid USA for social work students. Research on Social Work Practice.1-12.

19. Aakre JM, Lucksted A, Browning-McNee LA. Evaluation of Youth Mental Health First Aid USA: A program to assist young people in psychological distress. Psychological services. 2016;13(2):121.

20. Jorm AF, Kitchener BA, Sawyer M, Scales H, Cvetkovski S. Mental health first aid training for high school teachers: A cluster randomized trial. BMC Psychiatry. 2010;10:51. 21. Hart LM, Kelly CM, Kitchener BA, Jorm AF. teen Mental Health First Aid: A manual for young people helping their friends. Melbourne: Mental Health First Aid Australia; 2012.

22. Ross AM, Hart LM, Jorm AF, Kelly CM, Kitchener BK. Development of key messages for adolescents on providing basic mental health first aid to peers: a delphi consensus study. Early Intervention in Psychiatry. 2012;6(3):229-38. 
23. Hart LM, Mason RJ, Kelly CM, Cvetkovski S, Jorm AF. 'teen Mental Health First Aid': a description of the program and an initial evaluation. International Journal of Mental Health Systems. 2016;10(1):1-18.

24. Kessler RC, Andrews G, Colpe LJ, Hiripi E, Mroczek DK, Normand SLT, et al. Short screening scales to monitor population prevalences and trends in non-specific psychological distress. Psychological Medicine. 2002;32(06):959-76.

25. Kessler RC, Green JG, Gruber MIJ, Sampson NA, Bromet E, Cuitan M, et al. Screening for serious mental illness in the general population with the K6 screening scale: results from the WHO World Mental Health (WMH) survey initiative. International Journal of Methods in Psychiatric Research. 2010;19(0 1):4-22.

** 26. Hart LM, Morgan AJ, Rossetto A, Kelly CM, Mackinnon A, Jorm AF. Helping adolescents to better support their peers with a mental health problem: A clusterrandomised crossover trial of teen Mental Health First Aid. Australian \& New Zealand Journal of Psychiatry. 2018:Feb 8.

This article provides the most recent results on evaluations of teen Mental Health First Aid; using a RCT with 4 schools, 1942 students comparing outcomes with students who received a matched physical first aid training program.

27. Swartz KL, Musci RJ, Beaudry MB, Heley K, Miller L, Alfes C, et al. School-based curriculum to improve depression literacy among US secondary school students: a randomized effectiveness trial. American Journal of Public Health. 2017;107(12):1970-6. 28. Hart LM, Morgan AJ, Rossetto A, Kelly CM, Mackinnon A, Jorm AF. teenAID a trial to evaluate teen Mental Health First Aid. ACTRN12614000061639 Unpublished data. 29. Carbonell DM, Reinherz HZ, Giaconia RM, Stashwick CK, Paradis AD, Beardslee WR. Adolescent protective factors promoting resilience in young adults at risk for depression. Child and Adolescent Social Work Journal. 2002;19(5):393-412. 
30. Schmidt ME, Bagwell CL. The protective role of friendships in overtly and relationally victimized boys and girls. Merrill-Palmer Quarterly. 2007;53(3):439-60. 31. Jorm AF. Mental health literacy: empowering the community to take action for better mental health. American Psychologist. 2011;online.

32. Burns JR, Rapee RM. Adolescent mental health literacy: Young people's knowledge of depression and help seeking. Journal of Adolescence. 2006;29(2):225-39.

33. Jorm AF, Kitchener BA. Noting a landmark achievement: Mental health first aid training reaches $1 \%$ of Australian adults. Australian New Zealand Journal of Psychiatry. 2011;45(10):808-13.

34. MHFA. Our Global Impact Melbourne, Australia: Mental Health First Aid Australia; 2017 [Available from: https://mhfa.com.au/our-impact/our-global-impact]. 